\title{
I-shaped microchannel array chip for parallel electrophoretic analyses
}

\author{
Akira Inoue, ${ }^{1,2}$ Toshiyuki Ito, ${ }^{1}$ Kimiko Makino, ${ }^{2}$ Kazuo Hosokawa, ${ }^{* 1}$ and Mizuo Maeda ${ }^{1}$ \\ ${ }^{1}$ Bioengineering Laboratory, RIKEN, 2-1 Hirosawa, Wako, Saitama 351-0198, Japan \\ ${ }^{2}$ Faculty of Pharmaceutical Sciences, Tokyo University of Science, 2641 Yamazaki, Noda, \\ Chiba 278-8510, Japan \\ *E-mail: k-hoso@riken.go.jp

\section{Supporting Information}

1. Measurements of fluid displacements on PDMS-PMMA hybrid microchips.

2. Separation of dsDNA with a conventional cross injection method. 


\section{Measurements of fluid displacements on PDMS-PMMA hybrid microchips.}

Objective. The below experiments were carried out to provide an additional evidence for the hypothetical mechanism of the random bulk flow, which is discussed in the main text (Solution Filling in Results and Discussion section).

Methods. Design of the microchannel is shown in Figure S1a. Seven microchannels were fabricated on a surface of a PDMS part with the same molding technique as described in the main text. The PDMS part was reversibly bonded to a $2 \mathrm{~mm}$-thick poly(methyl methacrylate) (PMMA) plate (Acrylite, Mitsubishi Rayon; Tokyo, Japan). Two kinds of microchips were fabricated; they differ in structure of reservoirs. Type 1 microchip (Figure $\mathrm{S} 1 \mathrm{~b})$ has the reservoirs in the PMMA plate, and was fabricated as follows. 14 through-holes with a diameter of $2 \mathrm{~mm}$ were bored in the PMMA plate with a drilling machine. To make the inner surfaces of the reservoirs hydrophilic, the PMMA plate was treated with $\mathrm{O}_{2}$ plasma under the same conditions as those for PDMS described in the main text. During the plasma treatment, one side of the PMMA plate was protected with a blank PDMS which had reversibly been bonded to the surface. After the plasma treatment, the blank PDMS was peeled away, and the channel-molded PDMS was reversibly bonded to the protected (non-oxidized) side of the PMMA plate. Type 2 microchip (Figure S1c) has the reservoirs in the PDMS part, and was fabricated as follows. 14 through-holes with a diameter of $2 \mathrm{~mm}$ were punched in the channel-molded PDMS part using a metal pipe. The PDMS part was reversibly bonded to a blank PMMA plate. No plasma treatment was used for Type 2 microchip.

The microchip was degassed in a vacuum desiccator in the same way as described in the main text. After the degassing, $4 \mu \mathrm{L}$ of a mixture of $1.0 \mu \mathrm{M}$ fluorescently labeled oligonucleotide (denoted by "W(12)" in the main text) and 5\% PDMA was dispensed into the reservoir at one end of the microchannel, whereas $4 \mu \mathrm{L}$ of $5 \%$ PDMA was dispensed into the reservoir at the other end of the microchannel. Autonomous filling of the microchannel was observed using the microscope specified in the main text. Three minutes after the complete filling, a fluorescence image was taken.

Results and Discussion. Figure S1d is a photograph of Type 1 microchip. A typical fluorescence image is shown in Figure S1e. An image analysis software program (ImageJ $1.28 \mathrm{u}$, National Institutes of Health) was used to obtain the fluorescence intensity profile along the microchannel. We measured the position where the fluorescence intensity became 
the half of its maximum value. We defined the fluid displacement as the distance from the center of the stop valve to the position measured above, with the positive sign for the

(a)

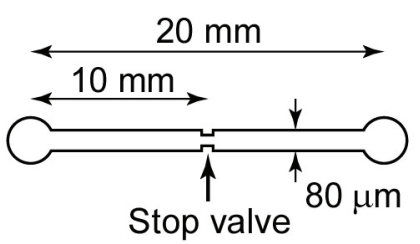

Depth: $25 \mu \mathrm{m}$

(b)

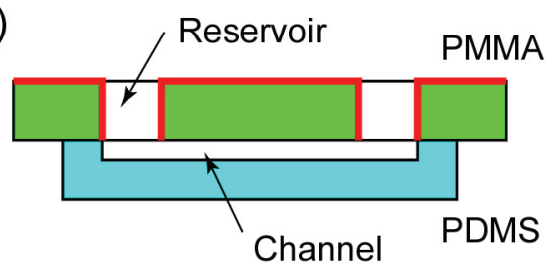

Oxidized surface

(c)

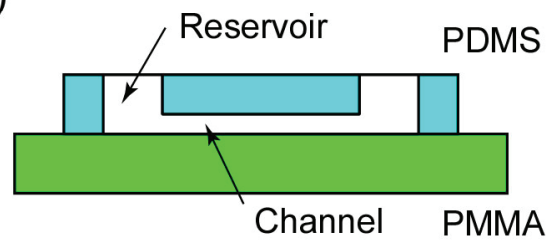

(d)

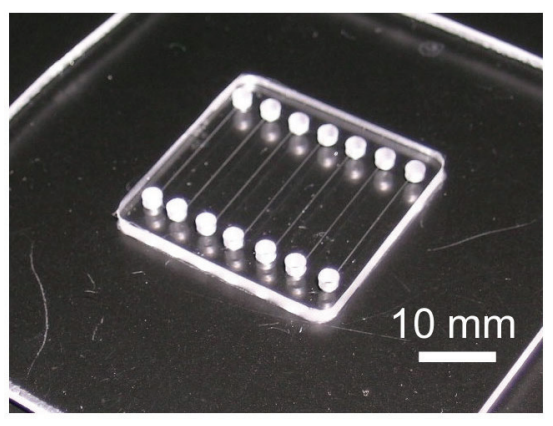

(e)

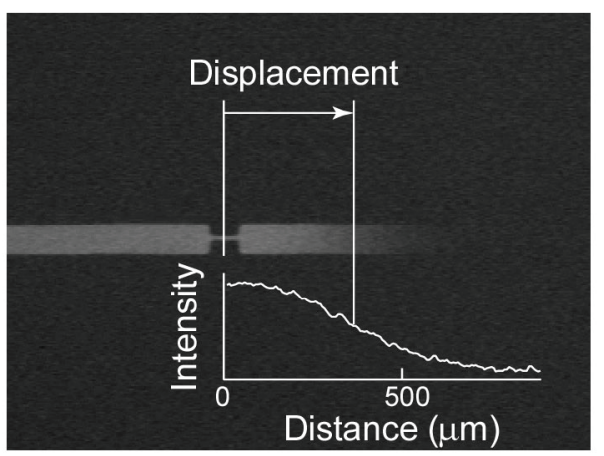

(f)

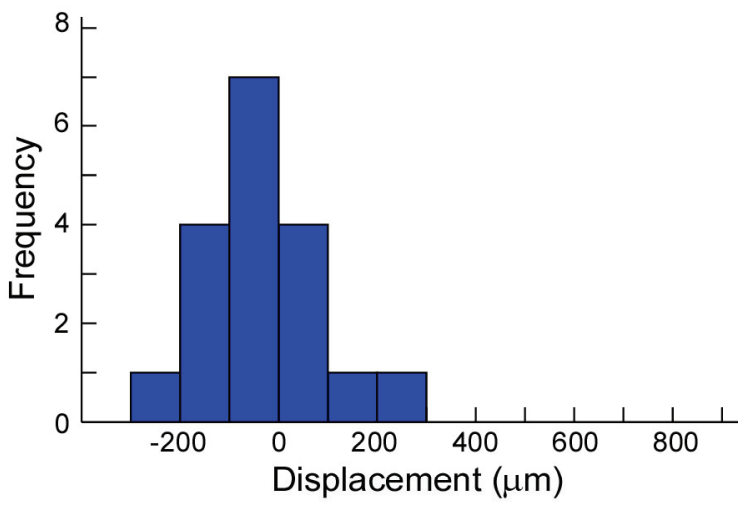

(g)

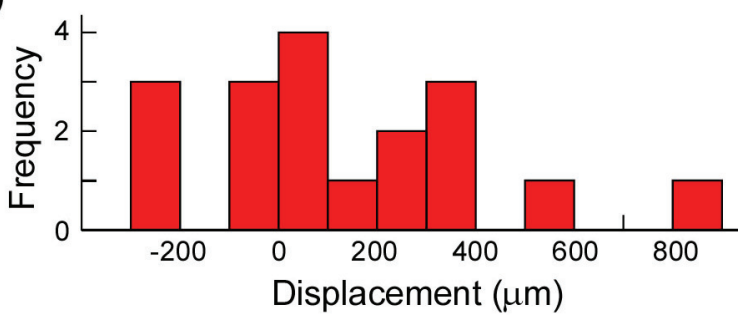

Figure S1. Measurements of fluid displacements on PDMS-PMMA microchips. (a) Design of the microchannel. The stop valve has the same geometry as described in Figure 1 in the main text. (b) Schematic cross-sectional view of Type 1 microchip. (c) Schematic cross-sectional view of Type 2 microchip. (d) A photograph of Type 1 microchip. (e) A typical fluorescence image showing the fluid displacement on Type 2 microchip. The inset shows the fluorescence intensity profile along the microchannel. (f) Histogram of the fluid displacements on Type 1 microchip. The average and the standard deviation of the displacements were $34 \mu \mathrm{m}$ and $118 \mu \mathrm{m}$, respectively $(N=18)$. $(\mathrm{g})$ Histogram of the fluid displacements on Type 2 microchip. The average and the standard deviation of the displacements were $123 \mu \mathrm{m}$ and $273 \mu \mathrm{m}$, respectively $(N=18)$. 
direction of the non-fluorescent solution.

Figures S1f and S1g are the histograms of the fluid displacements resulting from Type 1 and Type 2 microchips, respectively. The two histograms are significantly different; Type 1 microchip yielded much narrower distribution of the displacements than that Type 2; displacements larger than $300 \mu \mathrm{m}$ were observed in 5 cases out of 18 for Type 2 microchip, whereas no such cases were found for Type 1.

The above results strongly support the hypothetical mechanism of the random bulk flow (Figures $3 \mathrm{c}$ and $3 \mathrm{~d}$ in the main text). Namely, the surface property of the reservoirs is the main cause for the random bulk flow.

The above results also suggest a more practical strategy, to avoid the random bulk flow, than the current IMA chip, where the PDMS-made reservoirs were made hydrophilic with $\mathrm{O}_{2}$ plasma treatment. Unfortunately, $\mathrm{O}_{2}$ plasma-treated surfaces of PDMS are not permanently hydrophilic; they lose their hydrophilicity over time. ${ }^{1}$ In practical applications, the reservoirs should be permanently hydrophilic. For example, this could be done by fitting glass cylinders with an appropriate outer diameter into the through-holes of the PDMS part.

\section{Reference}

1. Kim, J.; Chaudhury, M. K.; Owen, M. J. J. Colloid Interface Sci. 2000, 226, 231-236. 


\section{Separation of dsDNA with a conventional cross injection method.}

Objective. The below experiments were carried out to compare the resolutions of dsDNA separations between the I-shaped microchannels and a conventional cross-shaped microchannel.

Methods. Design of the cross-shaped microchannel is shown in Figure S2a. The microchannel was fabricated on a surface of a PDMS part with the same molding technique as described in the main text. The reservoirs with a diameter of $2 \mathrm{~mm}$ were punched in the PDMS part using a metal pipe. The PDMS part was reversibly bonded to a $1 \mathrm{~mm}$-thick slide glass.

The microchannel was filled with the same 1.2\% HEC solution as used for the IMA chip. As a sample solution, the 100-1000-bp dsDNA ladder solution was prepared as described in the main text. We dispensed a $4 \mu \mathrm{L}$ aliquot of the sample solution into the sample reservoir (denoted by 1 in Figure S2a). As electrodes, Pt wires with a diameter of $0.5 \mathrm{~mm}$ were dipped into all the reservoirs. A home-made electric power supply was used for applying electric potentials to the electrodes. For sample injection, the potentials at reservoirs $1,2,3$, and 4 were kept at $-350 \mathrm{~V},+150 \mathrm{~V}, 0 \mathrm{~V}$, and $0 \mathrm{~V}$, respectively, for $90 \mathrm{~s}$. For separation, they were switched to $0 \mathrm{~V}, 0 \mathrm{~V},-100 \mathrm{~V}$, and $0 \mathrm{~V}$, respectively. The electrophoresis was carried out at room temperature $\left(\sim 25^{\circ} \mathrm{C}\right)$. The detection setup has been described in the main text. We set a detection point at $5 \mathrm{~mm}$ downstream from the injection point (the intersection). Four independent experiments were carried out.

Results and Discussion. A representative electropherogram is shown in Figure S2b. The resolution values were calculated, and have been compared with those of the IMA chip and other published methods in Table 1 in the main text. 
(a)

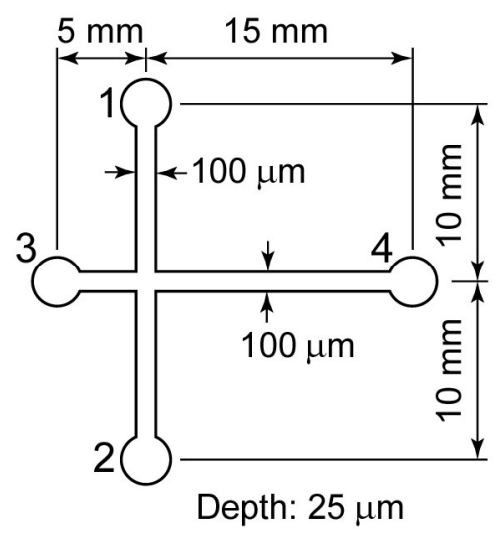

(b)

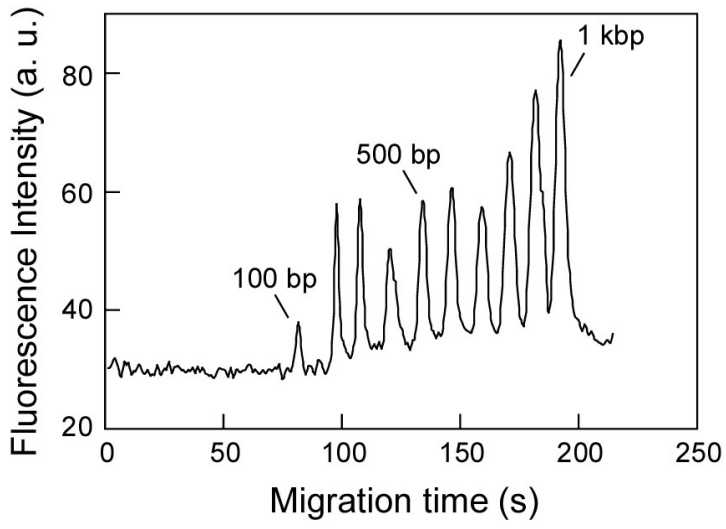

Figure S2. Separation of 100-1000-bp dsDNA ladder fragments in a conventional cross-shaped microchannel. (a) Design of the microchannel. (b) A representative electropherogram. Electric field, $50 \mathrm{~V} / \mathrm{cm}$; effective separation length, $5 \mathrm{~mm}$; buffer, $1 \times \mathrm{TBE}(\mathrm{pH} 8.0)$; polymer matrix, $1.2 \% \mathrm{HEC}$, sample concentration, $10 \mu \mathrm{g} / \mathrm{mL}$; label, SYBR Gold; $4 \times$ objective lens; temperature, $\sim 25^{\circ} \mathrm{C}$. 